\title{
Stable interactions in higher derivative field theories of derived type
}

\author{
V. A. Abakumova* and S. L. Lyakhovich ${ }^{\dagger}$ \\ Physics Faculty, Tomsk State University, Tomsk 634050, Russia \\ D. S. Kaparulin \\ Physics Faculty, Tomsk State University, Tomsk 634050, Russia \\ and Lebedev Institute of Physics, Leninsky Avenue 53, Moscow 119991, Russia
}

(Received 22 December 2018; published 28 February 2019)

\begin{abstract}
We consider the general higher derivative field theories of derived type. At free level, the wave operator of derived-type theory is a polynomial of the order $n \geq 2$ of another operator $W$ which is of the lower order. Every symmetry of $W$ gives rise to the series of independent higher order symmetries of the field equations of derived system. In its turn, these symmetries give rise to the series of independent conserved quantities. In particular, the translation invariance of operator $W$ results in the series of conserved tensors of the derived theory. The series involves $n$ independent conserved tensors including canonical energymomentum. Even if the canonical energy is unbounded, the other conserved tensors in the series can be bounded, that will make the dynamics stable. The general procedure is worked out to switch on the interactions such that the stability persists beyond the free level. The stable interaction vertices are inevitably non-Lagrangian. The stable theory, however, can admit consistent quantization. The general construction is exemplified by the order $N$ extension of Chern-Simons coupled to the Pais-Uhlenbeck-type higher derivative complex scalar field.
\end{abstract}

DOI: 10.1103/PhysRevD.99.045020

\section{INTRODUCTION}

The higher derivative field theories are notorious for the stability problems at interacting level, and also in quantum theory, see [1-4] for discussions and further references. The most frequently discussed higher derivative field theories are the modified models of gravity where the stability is an issue [5-7]. Among the modified gravity theories, $f(R)$ models (for review and further references see $[8,9]$ ) provide the best known example of stable nonlinear higher derivative field theory. Some other models with similar properties can be found in the Refs. [10-12]. The stability of this exceptional class of higher derivative theories is related to the fact that the canonical energy is bounded because of strong second class constraints. For discussion of stability in various nonlinear higher derivative mechanical models we refer to [13-17] and references therein.

In this paper, we consider a certain class of higher derivative models which we call derived theories. At free

\footnotetext{
*abakumova@phys.tsu.ru

†sl1@phys.tsu.ru

${ }^{\ddagger}$ dsc@phys.tsu.ru
}

Published by the American Physical Society under the terms of the Creative Commons Attribution 4.0 International license. Further distribution of this work must maintain attribution to the author(s) and the published article's title, journal citation, and DOI. Funded by SCOAP ${ }^{3}$. level, the field equations of the derived theory are defined by the higher derivative wave operator $M$ being a polynomial of another differential operator $W$. The latter is supposed to be of the first or second order. As we demonstrate, this class of systems admits, under certain conditions, inclusion of stable interactions, and the stability persists at quantum level.

Many well-known higher derivative models fall into the class of derived theories. For example, the higher derivative scalar field of the Pais-Uhlenbeck type [1] is a derived system, where $W$ is the d'Alembert operator. Podolsky electrodynamics [18] is a derived system, with the wave operator being a second-order polynomial of Maxwell operator. The extended Chern-Simons [19] is a derived theory of the vector field in $3 d$ Minkowski space, with the wave operator being a third-order polynomial in the ChernSimons operator $* d$, where $*$ is the Hodge star operator and $d$ is the de Rham differential. The Podolsky and ChernSimons electrodynamics have been discussed for many years from various viewpoints, see [20-22] and references therein. In the conformal gravities in 4 and 6 dimensions $[23,24]$ the linearized equations of motion for spin 2 fields belong to the derived type. The stability is studied in these works for the small fluctuations in the vicinity of constant curvature backgrounds further extending earlier observation of the work [25]. With a special choice of boundary conditions, it is observed that the theory might be stable. 
In the present work, we follow a different idea being unrelated to the special boundary conditions.

The simplest derived-type field equations correspond to the wave operator being the second-order polynomial in another operator. This case has been studied in [26]. In this class of models, it turns out that at free level one can connect two different conserved quantities to the time-shift symmetry. One of these quantities is the canonical energy, while another one is a different independent integral of motion. If the second quantity is bounded, the theory will be stable at classical level, and the stability can be promoted to quantum level [26]. In the paper [27], a more general setup of the derived systems is studied when the wave operator is a polynomial of arbitrary finite order $n$ in another operator [see relation (6) in the present work]. Once the primary operator $W$ admits some symmetry, it can be connected to $n$ conserved quantities of the derived system. In particular, if one of the symmetries of $W$ is the homogeneity of time, then one gets $n$ independent conserved quantities, which includes the canonical energy. In this paper, we adopt a slightly more general setup for the derived systems aimed at relaxing restrictions on inclusion of stable interactions. The fields are divided into several subsets such that the wave operator in each subset is a polynomial in a certain operator. Unlike a more simple case studied in [27], the primary operators can be different for different subsets of fields, and the wave operators are defined by different polynomials for different fields. This more general setup at free level provides more flexibility for inclusion of stable interactions.

The stability of interactions in various particular types of higher derivative theories of derived type has been previously studied in [26-29]. The proper deformation method has been suggested in [30] to systematically include stable interactions in the derived theory which is stable at free level. The crucial ingredient of the proper deformation method is the Lagrange anchor. The Lagrange anchor has been first introduced in the work [31] to Becchi-RouetStora-Tyutin embed and quantize not necessarily Lagrangian dynamics. Later, it has been found [32] that the Lagrange anchor connects conserved quantities to symmetries for any system of field equations, be they Lagrangian or not. For the Lagrangian system the unit operator serves as the Lagrange anchor that establishes oneto-one correspondence between symmetries and conserved currents. In principle, the Lagrange anchor is not necessarily unique for given system of field equations. Once the field equations admit multiple Lagrange anchors, the same symmetry can be connected to different conserved quantities. As is noticed in the paper [26], even the simplest derived system, with the wave operator $M$ being the second-order polynomial of another differential operator $W$, admits two different Lagrange anchors. This explains the existence of one more conserved quantity connected to time-independence besides the canonical energy. Given the
Lagrange anchor, the general method of [30] allows one to consistently include the interactions into field equations of motion deforming the conserved quantities connected with the symmetries by the anchor. If the anchor connects the symmetry with the bounded quantity, the system remains stable upon inclusion of interaction by the proper deformation scheme of [30].

In this paper, we provide a more simple scheme for inclusion stable interactions in a wider class of derived theories, skipping to explicitly employ the Lagrange anchor. The basic idea is that we have two subsets of fields such that the wave operator in each subset is a polynomial in certain primary operator. The primary operators are assumed to be Poincaré invariant. One of the primary wave operators is supposed to be gauge invariant. We also assume that the primary theories admit appropriate covariant interaction vertex consistent with the gauge symmetry. To construct the stable interactions between two derived theories, we identify the series of conserved tensors at free level such that every item is connected to the space-time translation symmetry. Some of the conserved tensors can have bounded 00-component, while the other ones are unbounded. Then, we seek for the interactions in the derived theories such that generalize couplings between primary models and keep the appropriate bounded quantity conserved at interacting level. If the theory admits bounded conserved quantity, the stability will persist at the interacting level once it is constructed by this method. The proposed construction can reproduce all the previously known stable interactions in higher derivative systems of derived type [26-28] and we also add a new example in this paper, to illustrate the method.

As the illustration of the general method described in the paper, we construct the consistent and stable interaction between the order $N$ extension of Chern-Simons and order $2 n$ Pais-Uhlenbeck-type higher derivative complex scalar field. These models have been discussed earlier at free level in the papers [26-29] for some specific orders $n$ and $N$. In this paper, the $N+n$-parameter series of conserved second-rank tensors is constructed for the free model being connected to the translation invariance. Also $n$-parameter series of conserved currents is identified to be connected to a single $U(1)$-symmetry of the scalar. There are bounded quantities among the conserved observables that make the theory stable at free level, while the canonical energy is unbounded. Nonminimal gauge invariant couplings are identified such that the higher derivative theory remains stable at interacting level. The stable interaction vertices are inevitably non-Lagrangian. That does not necessarily obstruct quantization as we explain in the conclusion.

The article is organized as follows. In the next section, we define the free derived theories and elaborate on the series of symmetries and conserved quantities connected to each symmetry of the primary wave operator $W$. In Sec. III, we identify the gauge invariant couplings such that provide 
conservation of any fixed representative of the series of conserved quantities of the derived-type free system. In Sec. IV, we consider interactions between the higher derivative complex scalar field and higher derivative extension of Chern-Simons. Following general pattern of the previous section, we explicitly construct the conserved quantities and keep track of the stability making use of the bounded integrals of motion. In the conclusion, we discuss the results.

\section{DERIVED-TYPE THEORIES, HIGHER SYMMETRIES, AND CONSERVATION LAWS}

Consider a set of fields $\phi^{a}$ in $d$-dimensional Minkowski space with local coordinates $x^{\mu}, \mu=0, \ldots, d-1$. We systematically use the DeWitt condensed notation [33] in this paper. The fields are labeled by the condensed indices $a, b, \ldots$ Every condensed index accommodates all the vector, tensor, spinor, isotopic, etc. indices and the space-time coordinates. Summation in the condensed indices implies integration in $x^{\mu}$. In this notation, the linear differential operators are represented by matrices with condensed indices. We assume that the theory admits a constant metric which is used to raise and lower multiindices. In this way, every linear operator has the quadric form. These quadratic forms correspond to the local functionals which are bilinear in the fields. We also imply that the fields vanish at the infinity, so if the quadratic form vanishes in the condensed notation, this means that the corresponding local functional is the integral of total divergence.

In the condensed notation, any system of linear field equations reads:

$$
M_{a b} \phi^{b}=0,
$$

where $M_{a b}$ is the integral kernel of matrix differential operator. For the sake of simplicity, we assume that the matrix $M_{a b}$ is square, so that the number of equations in each space-time point coincides with the number of fields. In this class of theories, $M_{a b}$ is usually called the wave operator. The formal adjoint of the wave operator is defined by

$$
M_{a b}^{\dagger}=M_{b a} .
$$

The field equations are variational whenever $M^{\dagger}=M$, in which case the action functional $S[\phi]$ reads

$$
S[\phi]=\frac{1}{2}\langle\phi, M \phi\rangle,
$$

where the brackets $\langle$,$\rangle denote the natural pairing between$ the fields,

$$
\langle\phi, M \phi\rangle \equiv \phi^{a}(M \phi)_{a}, \quad(M \phi)_{a} \equiv M_{a b} \phi^{b},
$$

and summation is implied over the repeated multi-indices $a$ when they stand at different levels. If $M_{*}^{\dagger}=-M_{*}$ for some other operator $M_{*}$, its diagonal elements are total divergencies,

$$
\int d^{d} x \partial_{\mu} j^{\mu}(\phi)=\left\langle\phi, M_{*} \phi\right\rangle, \quad \forall \phi .
$$

Once the expression $M_{*} \phi$ vanishes on-shell (1), the latter formula establishes the relation between conserved currents and anti-self-adjoint operators.

We say that the variational theory (1) is of the derivedtype if the wave operator is a finite-order polynomial of another self-adjoint operator $W_{b}^{a}$, i.e.,

$$
\begin{aligned}
M(\alpha ; W) & =\sum_{p=0}^{n} \alpha_{p} W^{p}, \\
\left(W^{p}\right)^{a}{ }_{b} & =W^{a}{ }_{{ }_{1}} W^{c_{1}}{ }_{c_{2}} \ldots W^{c_{p-1}} b, \\
p & =2, \ldots, n, \quad W^{\dagger}=W,
\end{aligned}
$$

where all the multi-indices are raised and lowered by the metric, and $\alpha_{p}, p=0, \ldots, n$, are some real constants. The order of the polynomial is assumed to be irreducible, so the coefficient at the highest order is nonzero, $\alpha_{n} \neq 0$. In accordance with the definition, each derived theory is defined by two ingredients: the self-adjoint operator $W^{a}{ }_{b}$ and finite-order polynomial,

$$
M(\alpha ; z)=\sum_{p=0}^{n} \alpha_{p} z^{p},
$$

with $z$ being formal complex-valued variable. We call $M(\alpha ; z)$ the characteristic polynomial, while $W_{b}^{a}$ is referred to as the primary wave operator. Being considered in itself, the primary operator defines the primary free field theory,

$$
W_{a b} \phi^{b}=0
$$

As $W_{a b}$ is self-adjoint, the primary theory is variational. In our article, we mostly deal with the class of theories, where the primary wave operator does not involve higher derivatives. In this setting, the higher derivative derived model (1), (6) can be considered as originating from the lower order primary theory (8).

In this paper, we consider the linear operator $X^{a}{ }_{b}$ as a symmetry ${ }^{1}$ of linear equations (1) if it is interchangeable with the wave operator of theory in the following sense:

\footnotetext{
${ }^{1}$ The notion of symmetry can be understood in various ways. In this section, we provide the simple non-rigorous understanding, which is sufficient for the purposes of this work.
} 


$$
\begin{aligned}
& {[X, M]_{b}^{a}=Y_{c}^{a} M^{c}{ }_{b},} \\
& {[X, M]_{b}^{a}=X_{c}^{a}{ }_{c} M_{b}^{c}-M_{c}^{a} X_{b}^{c},}
\end{aligned}
$$

with $Y^{a}{ }_{c}$ being some other operator. Once this relation is valid, the operator $X^{a}{ }_{b}$ defines the linear transformation of the fields such that leaves the mass shell (1) intact,

$$
\begin{aligned}
\delta_{\xi}(M \phi)^{a} & =\xi(X+Y)^{a}{ }_{b}(M \phi)^{b} \approx 0, \\
\delta_{\xi} \phi^{a} & =\xi X^{a}{ }_{b} \phi^{b},
\end{aligned}
$$

where $\xi$ is the transformation parameter, being some constant. The sign $\approx$ means equality modulo equations $(1)$. Each theory admits the trivial symmetries that read

$$
X_{b}^{a}=\tilde{X}_{c}^{a} M_{b}^{c}, \quad Y_{b}^{a}=[\tilde{X}, M]_{b}^{a},
$$

where $\tilde{X}_{b}^{a}$ can be any operator. The corresponding transformations of fields vanish on-shell and do not contain any valuable information about the dynamics of model. We systematically ignore the trivial symmetries, considering any symmetry modulo trivial one.

The nontrivial symmetries of linear system are known to form an associative algebra. The multiplication operation is just a composition of operators. It is easy to verify the latter fact: for each pair of symmetries $\left(X_{1}\right)^{a}{ }_{b},\left(X_{2}\right)^{a}{ }_{b}$, we get

$$
\begin{aligned}
{\left[X_{1} X_{2}, M\right]^{a}{ }_{b} } & =\left(X_{1}\right)^{a}{ }_{c}\left[X_{2}, M\right]^{c}{ }_{b}+\left[X_{1}, M\right]^{a}{ }_{c}\left(X_{2}\right)^{c}{ }_{b} \\
& =\left(\left(Y_{1}\right)^{a}{ }_{d}\left(X_{2}\right)^{d}{ }_{c}+\left(X_{1}\right)^{a}{ }_{d}\left(Y_{2}\right)^{d}{ }_{c}+\left(Y_{2}\right)^{a}{ }_{c}\right) M^{c}{ }_{b} .
\end{aligned}
$$

The elements of associative algebra that commute with the wave operator form a subalgebra. In this paper, we mostly consider this subalgebra as it is connected to conserved quantities.

Now, consider the derived equations (1), (6). Assume that the primary model (8) has the symmetry $X^{a}{ }_{b}$ such that commutes with the primary wave operator,

$$
[X, W]=0 .
$$

Even if the primary theory admits a single symmetry, the associative algebra of symmetries of derived theory has the two natural generators- $X$ and $W$-that commute with each other,

$$
[X, M]=[W, M]=0 .
$$

By composing the primary symmetry $X$ with the degree of the primary wave operator $W$ we get the symmetry of the derived equations (6):

$$
\begin{aligned}
{\left[X_{p}, M\right] } & =0, \\
\left(X_{p}\right)_{b}^{a} & =X_{c}^{a}\left(W^{p}\right)_{b}^{c}, \quad p=0, \ldots, n-1 .
\end{aligned}
$$

Only the terms with $p=0, \ldots, n-1$ are relevant, because the higher powers of primary operator can be absorbed by the wave operator (6). In this way, for $p \geq n$, the symmetry reduces on-shell to the symmetry with $p<n$. The generators $X_{p}$ can be assembled into the $n$-parameter series of derived symmetries of derived model,

$$
X_{b}^{a}(\beta)=\sum_{p=0}^{n-1} \beta_{p}\left(X_{p}\right)^{a}{ }_{b},
$$

with $\beta_{p}, p=0, \ldots, n-1$, being real numbers. As is seen, all the representatives of the series originate from one and the same symmetry $X$ of primary model.

The symmetry (9) preserves the action functional (3) if its operator is anti-self-adjoint and commutes with the wave operator,

$$
[X, M]=0, \quad X^{\dagger}=-X .
$$

The corresponding conserved current $j^{\mu}(\phi)$, being quadratic in the fields, is defined by the condition

$$
\int d^{d} x \partial_{\mu} j^{\mu}(\phi)=\langle\phi, X M \phi\rangle
$$

This formula represents the Noetherian relationship between symmetries and conservation laws. In the class of derived theories, a single symmetry (13) of primary model (8) determines the series of derived symmetries (16). The associated $n$-parameter series of conserved currents reads

$$
\begin{aligned}
j^{\mu}(\beta) & =\sum_{p=0}^{n-1} \beta_{p} j_{p}{ }^{\mu}(\phi), \\
\int d^{d} x \partial_{\mu} j_{p}{ }^{\mu}(\phi) & =\left\langle\phi, X_{p} M \phi\right\rangle,
\end{aligned}
$$

where the quantities $j_{p}{ }^{\mu}, p=0, \ldots, n-1$, come from symmetries (15). In particular, $j_{0}{ }^{\mu}$ represents the Noether conserved current for the symmetry (13) of primary model, while $j_{p}{ }^{\mu}, p=1, \ldots, n-1$, are other quantities.

The simplest possible symmetry of the free field theory is the translation invariance. The translation generators $\partial_{\mu}$ are automatically anti-self-adjoint and they commute with the primary wave operator (8),

$$
\left[\partial^{\mu}, W\right]=0 .
$$

Once the primary wave operator is translation invariant, the derived theory enjoy $n$-parameter series (16) of derived symmetries originating from this invariance: 


$$
\begin{aligned}
X_{b}^{\mu a}(\beta) & =\sum_{p=0}^{n-1} \beta_{p}\left(X_{p}\right)^{\mu a}{ }_{b}, \\
\left(X_{p}\right)^{\mu a}{ }_{b} & =\left(\partial^{\mu} W^{p}\right)^{a}{ }_{b} .
\end{aligned}
$$

Each of these symmetries preserves the action (3) of the derived model (1), (6). The corresponding conserved currents (19) constitute the series of second-rank energymomentum tensors

$$
\begin{aligned}
\Theta^{\mu \nu}(\beta) & =\sum_{p=0}^{n-1} \beta_{p} T_{p}{ }^{\mu \nu}(\phi), \\
\partial_{\nu} T_{p}{ }^{\mu \nu}(\phi) & =\left\langle\phi, \partial^{\mu} W^{p} M \phi\right\rangle .
\end{aligned}
$$

This series includes the canonical energy-momentum as $T_{0}{ }^{\mu \nu}(\phi)$, while $T_{p}{ }^{\mu \nu}, p=1, \ldots, n-1$, are different conserved tensors associated with space-time translation invariance of the model. In this way, the translation invariance of the derived-type field theory results in the series of the conserved tensors.

As the stability of higher derivative model is concerned, the 00-component of the conserved tensor is of interest. The 00 -component of tensor (22) reads

$$
\Theta^{00}(\beta)=\sum_{p=0}^{n-1} \beta_{p} T_{p}{ }^{00}(\phi) .
$$

This expression is given by the sum of canonical energy $T_{0}{ }^{00}$ and the other contributions $T_{p}{ }^{00}, p=1, \ldots, n-1$. Even if the canonical energy is unbounded due to higher derivatives, the quantity (23) can define bounded conserved charge. The bounded conserved quantity, if it exists, stabilizes classical dynamics of derived model (1), (6) at free level.

The entries of conserved current series (19) [or conserved tensor series (22)] are independent in general, even though it is not a theorem. This fact is supported by the following observations: (i) the quantities $j_{p}{ }^{\mu}$ are bilinear forms in the fields $\phi^{a}$ and their space-time derivatives, and (ii) the total number of derivatives involved in $j_{p}{ }^{\mu}$ increases with $p$. Once the symmetry $X$ and primary operator $W$ are the matrix differential operators of orders $n_{X}$ and $n_{W}$, the conserved current $j_{p}{ }^{\mu}$ involves at most

$$
(p+n) n_{W}+n_{X}-1
$$

derivatives of fields. The term with the highest number of derivatives contributes to the conserved quantity, and it is on-shell nontrivial at least in the case without gauge symmetry and constraints. Applying this argument to $j_{n-1}{ }^{\mu}$, we conclude that the highest order term cannot come from the linear combination of other currents with the lower number of derivatives. Thus, $j_{n-1}{ }^{\mu}$ is an independent conserved quantity. Proceeding with the same argument to $j_{n-2}{ }^{\mu}, j_{n-3}{ }^{\mu}, \ldots, j_{0}{ }^{\mu}$, we conclude that all these quantities are not functions of each other. The maximal number of independent entries in the conserved current series (19) equals to the order $n$ of characteristic polynomial (7). In the higher derivative scalar field model the maximal number of independent conserved quantities is connected with the space-time translation invariance [26]. In gauge theories, some generators of series (19) can be on-shell trivial. For example, the extended Chern-Simons model of order $n$ admits $n-1$ independent conserved tensors (22) [27]. All the above applies to free theories. At the nonlinear level, the procedure of inclusion of interaction is sensitive to the choice of representative in the conserved tensor series (19) which is conserved at the interacting level. This motivates us to work with the full series of conserved quantities, even if some of its generators are dependent.

The discussion above does not address the gauge symmetries. Now we explain how the gauge symmetry is accounted for in the field theories of derived type. The wave operator may have a null-vector,

$$
M_{b}^{a} R_{\alpha}^{b}=0 .
$$

In this case, the derived theory (1), (6) admits the gauge transformation such that preserves the mass shell,

$$
\delta_{\varepsilon}\left(M_{a b} \phi^{b}\right)=0, \quad \delta_{\varepsilon} \phi^{a}=R_{\alpha}^{a} \varepsilon^{\alpha},
$$

where $\varepsilon^{\alpha}$ are the gauge parameters, being arbitrary functions of space-time coordinates. The operator $R^{a}{ }_{\alpha}$ is the gauge symmetry generator. In the present work, we consider the class of derived models whose gauge symmetries come from null-vectors of primary model, i.e.,

$$
M_{b}^{a} R_{\alpha}^{b}=0 \Leftrightarrow W^{a}{ }_{b}{ }_{\alpha}^{b}=0 .
$$

This property is not automatically satisfied for general derived theory, so this is an additional assumption. The Podolsky's electrodynamics and the extended ChernSimons are consistent with this assumption, for example.

\section{CONSISTENT INTERACTIONS OF DERIVED MODELS}

In this section, we construct the class of consistent interactions between two Poincaré invariant derived theories, with one of them being gauge. At free level, these theories admit series of conserved energy-momentum tensors. We construct the interaction such that provides conservation of the deformation of certain representative of energy-momentum tensor series. In so doing, we admit not necessarily Lagrangian vertices. ${ }^{2}$ To solve the problem we

\footnotetext{
${ }^{2}$ For general problems of inclusion of consistent interactions between not necessarily Lagrangian theories see [34].
} 
proceed from the assumption that the two primary theories admit consistent interaction vertex. Then, we seek for the way to lift this vertex to the level of derived theory in such a way that does not spoil the stability of the free derived theory.

Consider two subsets of fields $\phi^{a}, \Phi^{i}$ on $d$-dimensional Minkowski space, with the multi-indices $a, i$ labeling the fields. The primary operators are denoted by $W_{a b}$ and $\mathcal{W}_{i j}$, so the primary field equations read

$$
W_{a b} \phi^{b}=0, \quad \mathcal{W}_{i j} \Phi^{j}=0 .
$$

These equations are variational, with the action functional being the sum of actions (3) of the fields $\phi^{a}$ and $\Phi^{i}$. The primary operators are assumed to be invariant under the space-time translations,

$$
\left[\partial^{\mu}, W\right]=\left[\partial^{\mu}, \mathcal{W}\right]=0 .
$$

The Poincaré symmetry implies the existence of conserved energy-momentum tensor

$$
\Theta^{\mu \nu}(\phi, \Phi)=T^{\mu \nu}(\phi)+T^{\mu \nu}(\Phi),
$$

where $T^{\mu \nu}(\phi)$ and $T^{\mu \nu}(\Phi)$ denote the contributions of free fields $\phi$ and $\Phi$. The quantity $\Theta^{\mu \nu}$ is determined by the formula

$$
\int d^{d} x \partial_{\nu} \Theta^{\mu \nu}(\phi, \Phi)=\left\langle\phi, \partial^{\mu} W \phi\right\rangle+\left\langle\Phi, \partial^{\mu} \mathcal{W} \Phi\right\rangle .
$$

The theory of the field $\Phi^{i}$ is assumed to be gauge invariant. The gauge symmetry generator $R_{\alpha}^{i}$ of the field is the nullvector of the primary operator $\mathcal{W}_{i j}$,

$$
\mathcal{W}_{i j} R^{j}{ }_{\alpha}=0,
$$

see Eq. (25). The corresponding gauge transformation reads

$$
\delta_{\varepsilon} \Phi^{i}=R_{\alpha}^{i} \varepsilon^{\alpha}, \quad \delta_{\varepsilon} \phi^{a}=0,
$$

where $\varepsilon^{\alpha}$ are gauge parameters. We do not allow gauge freedom for the fields $\phi^{a}$.

We assume that there exists a variational interaction vertex between primary models such that (i) the gauge transformation (33) is preserved at the interacting level and (ii) the action functional is at most quadratic in $\phi$. The most general action such that meets these requirements has the form

$S[\phi, \Phi]=\frac{1}{2}\left(W_{a b}+\Gamma_{a b}(\beta \Phi)\right) \phi^{a} \phi^{b}+\frac{1}{2} \mathcal{W}_{i j} \Phi^{i} \Phi^{j}$,

where the operator of vertex $\Gamma_{a b}(\beta \Phi)$ is a function of quantity $\beta \Phi$, and $\beta$ is the coupling constant, which can be arbitrary real number. We assume that $\Gamma_{a b}(\beta \Phi)$ is a finiteorder polynomial in $\Phi^{i}$, i.e.,

$$
\Gamma_{a b}(\beta \Phi)=\sum_{k=1}^{k_{\max }} \beta^{k} \Gamma_{a b}^{(k)}(\Phi), \quad k_{\max }<+\infty,
$$

with $\Gamma^{(1)}{ }_{a b}, \Gamma^{(2)}{ }_{a b}, \ldots$ being linear, quadratic etc. in the field $\Phi$. The expression $\beta^{k}$ means the $k$ th power of $\beta$. The presence of such decomposition ensures that the equations of motion of the model are finite-order polynomials in the coupling constant $\beta$. The Lagrange equations for the action functional (34) read

$$
\begin{aligned}
\partial_{a} S & \equiv W_{a b}(\beta \Phi) \phi^{b}=0, \\
\partial_{i} S & \equiv \mathcal{W}_{i j} \Phi^{j}+\beta J_{i}(\phi, \beta \Phi)=0,
\end{aligned}
$$

where

$$
\begin{aligned}
W_{a b}(\beta \Phi) & =W_{a b}+\Gamma_{a b}(\beta \Phi), \\
J_{i}(\phi, \beta \Phi) & =\partial_{i} \Gamma_{a b}(\beta \Phi) \phi^{a} \phi^{b},
\end{aligned}
$$

and the derivatives $\partial_{a}, \partial_{i}$ are understood as variational in $\phi^{a}, \Phi^{i}$. As is seen, in the model with interaction, the operator of vertex $\Gamma_{a b}(\beta \Phi)$ is added to free equations of originally nongauge field $\phi^{a}$, while the gauge field theory get additional current-like contribution $J_{i}(\phi, \beta \Phi)$. This structure of nonlinear theory is typical for lower spin fields. For example, it includes the minimal coupling between the electromagnetic field and the charged matter fields.

The infinitesimal gauge transformation of the action functional (34) reads

$$
\delta_{\varepsilon} \phi^{a}=\beta R_{b \alpha}^{a} \phi^{b} \varepsilon^{\alpha}, \quad \delta_{\varepsilon} \Phi^{i}=R_{\alpha}^{i} \varepsilon^{\alpha},
$$

where $R_{\alpha}^{i}$ is the null-vector of primary operator $\mathcal{W}_{i j}(32)$, and $R_{b \alpha}^{a}$ is some field-independent structure function. We assume that it is antisymmetric in the indices $a b$,

$$
R_{a b \alpha}=-R_{b a \alpha} .
$$

The invariance of action (34) with respect to the gauge transformations (38) is equivalent to the condition

$$
\begin{aligned}
& (W+\Gamma(\beta \Phi))^{a}{ }_{c} R_{b \alpha}^{c}-R_{c \alpha}^{a}(W+\Gamma(\beta \Phi))^{c}{ }_{b} \\
& \quad+R_{\alpha}^{i} \partial_{i} \Gamma_{b}^{a}{ }_{b}(\beta \Phi)=0 .
\end{aligned}
$$

As the gauge symmetries and gauge identities of Lagrangian theories are connected by the Noether theorem, conditions (39), (40) determine the selection rule for the structure functions $\Gamma_{b}^{a}, R_{b \alpha}^{a}$. The meaning of these conditions is that the wave operator $W_{a b}(\beta \Phi)$ (37) is gauge invariant in the following sense: 


$$
\begin{aligned}
& {\left[W(\beta \Phi), R_{\alpha}\right]_{a b}+R_{\alpha}^{i} \partial_{i} \Gamma_{a b}(\beta \Phi)=0,} \\
& {\left[W(\beta \Phi), R_{\alpha}\right]_{a b}=W_{a c}(\beta \Phi) R_{b \alpha}^{c}-R_{a c \alpha} W_{b}^{c}(\beta \Phi), \quad \forall \beta .}
\end{aligned}
$$

The latter relation immediately ensures the gauge invariance of equations of motion of the field $\phi^{a}$.

The translation invariance of interacting theory (34) is understood in the following sense:

$$
\begin{aligned}
& {\left[W(\beta \Phi), \partial^{\mu}\right]_{a b}+\beta \partial^{\mu} \Phi^{i} \partial_{i} \Gamma_{a b}(\beta \Phi)=0,} \\
& {\left[W(\beta \Phi), \partial^{\mu}\right]_{a b}=W_{a b}(\beta \Phi) \partial^{\mu}-\partial^{\mu} W_{a b}(\beta \Phi), \quad \forall \beta .}
\end{aligned}
$$

Once this condition is met, the action functional (34) is preserved by the space-time translations. The energymomentum tensor of the nonlinear theory has the following structure:

$$
\Theta^{\mu \nu}(\phi, \Phi)=T^{\mu \nu}(\phi, \Phi)+T^{\mu \nu}(\Phi)
$$

The expression $T^{\mu \nu}(\phi, \Phi)$ is the gauge invariant extension of the tensor $T^{\mu \nu}(\phi)$ (30) of free theory, while $T^{\mu \nu}(\Phi)$ is the energy-momentum of free gauge field. The defining condition for the conserved tensor reads

$$
\int d^{d} x \partial_{\nu} \Theta^{\mu \nu}(\phi, \Phi)=\left\langle\phi, \partial^{\mu} W(\beta \Phi) \phi\right\rangle+\left\langle\Phi, \partial^{\mu} \mathcal{W} \Phi\right\rangle .
$$

The right-hand side (rhs) of this expression is a total divergence because $\partial^{\mu} W(\beta \Phi), \partial^{\mu} \mathcal{W}$ are anti-self-adjoint operators.

Let us explain the meaning of conditions (39), (40) and its impact on the structure of interactions. Assume that the gauge transformation of the gauge field $\Phi^{i}$ has a null-mode $\varepsilon=\bar{\varepsilon}(x)$ such that

$$
R_{\alpha}^{i} \bar{\varepsilon}^{\alpha}=0 .
$$

In this case, the action functional (34) is preserved by the following transformation:

$$
\delta_{\bar{\varepsilon}} \phi^{a}=\xi R_{b \alpha}^{a} \bar{\varepsilon}^{\alpha}, \quad \delta_{\bar{\varepsilon}} \Phi^{i}=0,
$$

with the parameter $\xi$ being a constant. In the free limit, this transformation corresponds to certain internal symmetry of the free model of the fields $\phi^{a}$, which localizes at the interacting level. This symmetry does not follow from the relativistic invariance of equations (28), so we have additional prerequisite for constriction of interaction at free level. As we deal with internal symmetry, this gives a restriction on the multiplet of fields $\phi^{a}$ that are involved into the interaction.

The currentlike term $J_{i}$ (37) originates from the internal symmetry (46). As a consequence of relations (39), (40), it is gauge invariant and satisfies gauge identity,

$$
\delta_{\varepsilon} J_{i}=0, \quad R_{\alpha}^{i} J_{i}+R_{b \alpha}^{a} \partial_{a} S \phi^{b}=0 .
$$

If the internal symmetry is the $U(1)$-transformation, and gauge generator $R_{\alpha}^{i}$ is gradient, the currentlike term literally corresponds to the current of the $U(1)$-charge,

$$
J_{i}=J_{\mu}(x),
$$

which meets condition (47). Once currentlike term $J_{i}$ is added to the equations of motion of the gauge field, and the primary operator $W$ is replaced by its gauge invariant extension $W(\beta \Phi)$, the nonlinear theory remains gauge invariant. This means that interactions (36) imitate the electromagneticlike couplings between gauge and matter fields.

Let us now turn to the details of inclusion of interactions between derived theories. The most general ansatz for two derived theories with the primary operators $W$ and $\mathcal{W}$ has the form

$$
M_{a b}(\alpha ; W) \phi^{b}=0, \quad \mathcal{M}_{i j}(A ; \mathcal{W}) \Phi^{j}=0 .
$$

The characteristic polynomials of derived models (49) are the most general of orders $n$ and $N$, respectively,

$$
M(\alpha ; z)=\sum_{p=0}^{n} \alpha_{p} z^{p}, \quad \mathcal{M}(A ; z)=\sum_{q=1}^{N} A_{q} z^{q}
$$

where $z$ is a formal complex-valued variable. The real numbers $\alpha_{p}, p=0, \ldots, n$, and $A_{q}, q=1, \ldots, N$, are model parameters that distinguish different theories in the considered class.

The derived theories (49) have the same space-time and gauge symmetries as primary models. In particular, models (49), (50) are invariant under gauge transformation (33). As for space-time translation, a single symmetry of primary theory induces the $n+N$-parameter series of derived symmetries such that

$$
\begin{aligned}
X_{b}^{\mu a}(\beta) & =\sum_{p=0}^{n-1} \beta_{p}\left(\partial^{\mu} W^{p}\right)^{a}{ }_{b}, \\
X_{j}^{\mu i}(B) & =\sum_{q=0}^{N-1} B_{q}\left(\partial^{\mu} \mathcal{W}^{q}\right)^{i}{ }_{j},
\end{aligned}
$$

where the real numbers $\beta_{p}, B_{q}$ are parameters. The corresponding set of the conserved quantities is given by the series of energy-momentum tensors

$$
\Theta^{\mu \nu}(\beta, B)=\sum_{p=0}^{n-1} \beta_{p} T_{p}{ }^{\mu \nu}(\phi)+\sum_{q=0}^{N-1} B_{q} T_{q}{ }^{\mu \nu}(\Phi),
$$


where the quantities $T_{p}{ }^{\mu \nu}(\phi), T_{q}{ }^{\mu \nu}(\Phi)$ are defined by the conditions

$$
\begin{aligned}
\int d^{d} x \partial_{\nu} \Theta^{\mu \nu}(\beta, B)= & \sum_{p=0}^{n-1} \beta_{p}\left\langle\phi, \partial^{\mu} W^{p} M \phi\right\rangle \\
& +\sum_{q=0}^{N-1} B_{q}\left\langle\Phi, \partial^{\mu} \mathcal{W}^{q} \mathcal{M} \Phi\right\rangle
\end{aligned}
$$

By construction, the canonical energy-momentum tensor of the model is included in the series as

$$
\Theta_{\mathrm{can}}^{\mu \nu}(\phi, \Phi)=T_{0}{ }^{\mu \nu}(\phi)+T_{0}{ }^{\mu \nu}(\Phi) .
$$

Even though the canonical energy-momentum is almost always unbounded due to higher derivatives, the other bounded quantities can present in the series. Free higher derivative theory can be stabilized by these quantities.

By interaction between two gauge models (49) we mean a formal deformation of free theory with coupling parameter $\beta$ such that the nonlinear equations of motion read

$$
\begin{gathered}
E_{a}(\beta) \equiv M_{a b} \phi^{b}+\sum_{k=1}^{\infty} \beta^{k} \Gamma^{(k)}{ }_{a}(\phi, \Phi)=0, \\
\mathcal{E}_{i}(\beta) \equiv \mathcal{M}_{i j} \Phi^{j}+\sum_{k=1}^{\infty} \beta^{k} \Gamma^{(k)}{ }_{i}(\phi, \Phi)=0 .
\end{gathered}
$$

The structure functions $\Gamma^{(k)}$ are assumed to have the homogeneity degree $k+1$ in the variables $\phi^{a}, \Phi^{i}$, so $\Gamma^{(1)}$ are quadratic in the fields, $\Gamma^{(2)}$ are cubic, and etc. No action principle is required for interacting theory, so nonlinear equations (55) can be non-Lagragian. We consider interaction (55) consistent if the number of gauge symmetries and gauge identities is preserved by coupling and at least one representative of the free series of energymomentum tensors (52) is still conserved at the nonlinear level.

The $n+N$-parameter series of interaction vertices for free equations (49) can be presented in the following form

$$
\begin{aligned}
E_{a} & \equiv \bar{M}_{a b}(\beta, B) \phi^{b}=0, \\
\mathcal{E}_{i} & \equiv \mathcal{M}_{i j} \Phi^{j}+\sum_{p=0}^{n-1} \beta_{p}\left(\bar{J}_{p}\right)_{i}(\beta, B)=0,
\end{aligned}
$$

where the notation is used:

$$
\begin{aligned}
\bar{M}_{a b}(\beta, B) & =\sum_{p=0}^{n} \alpha_{p} \bar{W}^{p}(\beta, B), \\
\left(\bar{J}_{p}\right)_{i}(\beta, B) & =\frac{1}{2} \partial_{i}\left\langle\phi, \bar{W}^{p}(\beta, B) \bar{M}(\beta, B) \phi\right\rangle,
\end{aligned}
$$

and the line above means that the gauge field $\Phi^{i}$ is replaced by more general expression:

$$
\begin{aligned}
& \bar{W}(\beta, B)=\left.W(\beta \Phi)\right|_{\beta \Phi=\bar{\Phi}(\beta, B)}, \\
& \bar{\Phi}^{i}(\beta, B)=\beta \Phi^{i}+\sum_{q=1}^{N-1} B_{q}\left(\mathcal{W}^{q} \Phi\right)^{i} .
\end{aligned}
$$

The coupling constants are real numbers $\beta, \beta_{p}, B_{q}$, $p=0, \ldots, n-1, q=1, \ldots, N-1$. Interactions (56) generalize couplings of the primary theory (36) in the following sense: the free wave operator $M$ of the fields $\phi^{a}$ is replaced by its gauge invariant extension $\bar{M}(\beta, B)$, and the series of current like terms $\left(\bar{J}_{p}\right)_{i}(\beta, B)$ is added to the free gauge equations.

The following facts ensure consistency of interaction (56): (i) the equations of motion are preserved by the gauge transformation (38), while transformation law for the equations of motion reads

$$
\delta_{\epsilon} E^{a}=\beta R_{b \alpha}^{a} E^{b} \varepsilon^{\alpha}, \quad \delta_{\varepsilon} \mathcal{E}^{i}=0
$$

(ii) there are the gauge identities between equations of motion,

$$
R_{\alpha}^{i} \mathcal{E}_{i}+\sum_{p=0}^{n-1} \beta_{p} R_{a b \alpha}\left(\bar{W}^{p}\right)_{c}^{b} E^{a} \phi^{c} \equiv 0
$$

(iii) the second-rank conserved tensor $\Theta^{\mu \nu}$ is defined by the condition

$$
\begin{aligned}
\int d^{d} x \partial_{\nu} \Theta^{\mu \nu}(\beta, B)= & \sum_{p=0}^{n-1} \beta_{p}\left\langle\phi, \partial^{\mu} \bar{W}^{p} E\right\rangle \\
& +\sum_{q=0}^{N-1} B_{q}\left\langle\Phi, \partial^{\mu} \mathcal{W}^{q} \mathcal{E}\right\rangle, \quad B_{0} \equiv \beta .
\end{aligned}
$$

The proof of relations (59), (60) uses identities

$$
\begin{aligned}
& \text { (a) } \delta_{\varepsilon}\left(\bar{W}^{p} \phi\right)^{a}=\beta R_{b \alpha}^{a}\left(\bar{W}^{p} \phi\right)^{b} \varepsilon^{\alpha} ; \\
& \text { (b) } R_{\alpha}^{i}\left(\bar{J}_{p}\right)_{i}+R_{a b \alpha}\left(\bar{W}^{p}\right)^{b}{ }_{c} E^{a} \phi^{c} \equiv 0 ; \\
& \text { (c) } \delta_{\varepsilon}\left(\bar{J}_{p}\right)_{i}=0,
\end{aligned}
$$

which hold for $p=0, \ldots, n-1$. We deduce these relations in the Appendix A. The conserved tensor $\boldsymbol{\Theta}^{\mu \nu}$ (61) is a deformation of a selected representative of free energymomentum series (53), whose parameters are defined by coupling constants. In Appendix B, we ensure the rhs of Eq. (61) is a total divergence and establish the structure of conserved quantity. 
Let us comment on the origin of interactions (56). The model (49) admits $n$-parameter series of gauge invariant currentlike terms

$$
\begin{aligned}
J_{i}(\beta) & =\sum_{p=0}^{n-1} \beta_{p}\left(J_{p}\right)_{i}(\beta), \\
\left(J_{p}\right)_{i}(\beta) & =\frac{1}{2} \partial_{i}\left\langle\phi, W^{p}(\beta \Phi) M(\beta \Phi) \phi\right\rangle .
\end{aligned}
$$

Once the primary wave operator $W$ is replaced by its gauge invariant extension $W(\beta \Phi)$, the inclusion of such currentlike terms preserves gauge invariance. This means that theory (56) with the gauge-invariant primary operator (37) and current-like term (63) is consistent. At this ways, we conclude that there is $n+1$-parameter series of interactions with coupling constants $\beta, \beta_{0}, \ldots, \beta_{n-1}$. To involve coupling constants $B_{q}$ into account, we perform the shift of the gauge field $\beta \Phi \mapsto \bar{\Phi}(\beta, B)$ (58). This step preserves consistency of interactions and inserts $N-1$ coupling parameters $B_{1}, \ldots, B_{N-1}$ into the field equations.

The nonlinear theory (56) is stable if the bounded representative of free energy-momentum tensor series (52) is still conserved at the interacting level. The latter requirement can be interpreted as the selection rule for admissible couplings. The derived theories, whose characteristic polynomial (7) has nondegenerate real roots, are usually stable at free level. ${ }^{3}$ This gives a good chance for existence of stable interactions between such models. The theories with multiple real or/and complex roots of the characteristic polynomial usually have the degrees of freedom whose dynamics is unbounded by any conserved quantity. These degrees of freedom are the true ghosts unless they are suppressed by constraints or gauged out. The stability of interactions in the models having no bounded conserved quantity at free level is a subtle issue, which we do not consider in the present paper.

The general representative in the class of interacting theories (56) is non-Lagrangian as the equations of motion are not given by the variational derivatives of any functional. The field equations are Lagrangian if the values of coupling parameters meet the condition

$$
\beta_{0}=\beta,
$$

and all other constants vanish. The action functional reads

$$
S=\frac{1}{2}(\langle\phi, M(\beta \Phi) \phi\rangle+\langle\Phi, \mathcal{M} \Phi\rangle) .
$$

The corresponding conserved quantity is the canonical energy-momentum tensor of the model. In the class of higher derivative derived equations, the variational interaction

\footnotetext{
${ }^{3}$ Several known examples confirm this observation [27,29,28], though it is not a theorem at the moment.
}

vertex usually suffers from the Ostrogradski instability. In contrast to Lagrangian interactions, the non-Lagrangian couplings can preserve bounded representative in the series of free conserved quantities (52). These couplings define the stable nonlinear theory.

Let us summarize the results of this section. Relations (56)-(58) provide a general receipt of construction of series of gauge invariant interaction vertices between two derived theories, if the gauge invariant coupling is known for primary models. The structure of interaction vertex resembles couplings between electromagnetic field and charged matter: the wave operator of originally non-gauge field is replaced by its gauge invariant extension, while the currentlike term is added to equations of motion of gauge field. If the characteristic polynomials of derived theories have the orders $n$ and $N$, there are $n+N$ coupling constants. Any specific choice of coupling constants selects certain representative in the series of free conserved energymomentum such that still conserves at the interacting level. If the free theory has bounded conserved quantities, it admits the stable interactions, though the stable couplings are non-Lagrangian.

\section{COUPLINGS BETWEEN EXTENDED CHERN-SIMONS AND HIGHER DERIVATIVE SCALAR}

Following the general pattern of the previous section, let us consider the interactions between the gauge vector field and the charged scalar. In this case, we have two subsets of fields on $3 d$ Minkowski space,

$$
\phi^{a}=(\operatorname{Re} \phi)(x)+i(\operatorname{Im} \phi)(x), \quad \Phi^{i}=\Phi^{\mu}(x) .
$$

The primary operators read

$$
W=m^{-2} \partial_{\mu} \partial^{\mu}, \quad \mathcal{W}_{\mu \nu}=m^{-1} \varepsilon_{\mu \rho \nu} \partial^{\rho}
$$

To make contact with the general scheme of previous section, we identify the d'Alembertian as the primary operator for the scalar. For the vector field, the ChernSimons operator serves as the primary operator. The constant $m>0$ has the dimension of mass. Here we use it to make the primary operators dimensionless. The primary equations of motion,

$$
m^{-2} \partial_{\mu} \partial^{\mu} \phi=0, \quad m^{-1} \varepsilon_{\mu \nu \rho} \partial^{\nu} \Phi^{\rho}=0,
$$

have the gauge symmetry (33), with the gauge generator for the vector field being gradient, $R_{\alpha}^{i} \equiv \partial^{\mu}$.

The primary models (68) admit obvious variational interaction vertex,

$$
S[\phi, \Phi]=\frac{1}{2} \int\left[\phi^{*} D_{\mu} D^{\mu} \phi+m \varepsilon_{\mu \nu \rho} \Phi^{\mu} \partial^{\nu} \Phi^{\rho}\right] d^{3} x,
$$


where $D_{\mu}$ is the covariant derivative,

$$
D_{\mu}(\beta) \phi=\left(\partial_{\mu}-i \beta \Phi_{\mu}\right) \phi
$$

For the complex conjugate field $\phi^{*}$, the covariant derivative is given by the complex conjugation of (70). The action functional (69) has form (34) with the operator of vertex,

$$
\begin{aligned}
\Gamma & \equiv D_{\mu} D^{\mu}-\partial_{\mu} \partial^{\mu} \\
& =\left(\partial_{\mu}-i \beta \Phi_{\mu}\right)\left(\partial^{\mu}-i \beta \Phi^{\mu}\right)-\partial_{\mu} \partial^{\mu},
\end{aligned}
$$

being the second-order polynomial in the coupling constant $\beta$. The Lagrange equations for the action functional (69) read

$$
\begin{aligned}
& \frac{\delta S}{\delta \Phi^{\mu}} \equiv m \varepsilon_{\mu \nu \rho} \partial^{\nu} \Phi^{\rho}+i \beta\left(\phi^{*} D_{\mu} \phi-\phi D_{\mu} \phi^{*}\right)=0, \\
& \frac{\delta S}{\delta \phi} \equiv \frac{1}{2} D_{\mu} D^{\mu} \phi^{*}=0 .
\end{aligned}
$$

These equations have form (36) with

$$
\begin{aligned}
& J_{i} \equiv J_{\mu}(\phi, \beta \Phi)=i\left(\phi^{*} D_{\mu} \phi-\phi D_{\mu} \phi^{*}\right), \\
& W(\beta \Phi)=D_{\mu} D^{\mu} .
\end{aligned}
$$

As the gauge field is a vector, the current-like term holds world index. As we can see, the quantity $J_{\mu}(\phi, \beta \Phi)$ is the charge current of complex scalar field. The infinitesimal gauge transformation (38) of the action functional (69) reads

$$
\delta \phi=i \beta \phi^{*} \varepsilon, \quad \delta \Phi^{\mu}=\partial^{\mu} \varepsilon .
$$

The canonical energy-momentum tensor (43) for the action (69) has the form

$$
\begin{gathered}
\boldsymbol{\Theta}_{\mu \nu}=F_{\mu} F_{\nu}-\frac{1}{2} \eta_{\mu \nu} F_{\lambda} F^{\lambda}+D_{\mu} \phi^{*} D_{\nu} \phi+D_{\nu} \phi^{*} D_{\mu} \phi \\
-\eta_{\mu \nu} D_{\lambda} \phi^{*} D^{\lambda} \phi, \quad F_{\mu} \equiv m \varepsilon_{\mu \nu \rho} \partial^{\nu} \Phi^{\rho} .
\end{gathered}
$$

It is obvious that its 00-component is bounded. So, the theory of interacting Chern-Simons and charged scalar is stable at the level of primary theories.

Now we consider interactions between two derived theories, whose primary operators are given by (67). The free equations (49) are chosen in the form

$$
\begin{aligned}
& M(\alpha ; W) \phi \equiv m^{2} \prod_{p=0}^{n-1}\left(m^{-2} \partial_{\mu} \partial^{\mu}+\alpha_{p}^{2}\right) \phi=0, \\
& \mathcal{M}_{\mu \nu}(A ; \mathcal{W}) \Phi^{\nu} \equiv \frac{m^{2}}{2} \sum_{q=1}^{N} A_{q}\left(\mathcal{W}^{q}\right)_{\mu \nu} \Phi^{\nu}=0 .
\end{aligned}
$$

Here we assume that all the roots of characteristic polynomial (7) for scalar field are different and positive. We ignore all the other options because they do not result to the stable theory at the free or interacting level. The extended covariant derivative $\bar{D}_{\mu}$ is defined as follows:

$\bar{D}_{\mu}(\beta, B) \phi=\left(\partial_{\mu}-i \sum_{q=0}^{N-1} B_{q}\left(\mathcal{W}^{q}\right)_{\mu \nu} \Phi^{\nu}\right) \phi, \quad B_{0} \equiv \beta$

The gauge invariant extension of the free wave operator $M(\alpha ; W)(76)$ reads

$\bar{M}(\alpha ; \bar{W}(\beta \Phi)) \phi \equiv m^{2} \prod_{p=0}^{n-1}\left(m^{-2} \bar{D}_{\mu} \bar{D}^{\mu}+\alpha_{p}^{2}\right) \phi=0$.

The series of currentlike terms (63) can be represented in the form

$$
\left(J_{p}\right)_{\mu}=i\left(\phi^{*(p)} \bar{D}_{\mu} \phi^{(p)}-\phi^{(p)} \bar{D}_{\mu} \phi^{*(p)}\right), \quad p=0, \ldots, n-1,
$$

where the notation is used: ${ }^{4}$

$\phi^{(p)}=\prod_{\substack{p^{\prime}=0 \\ p^{\prime} \neq p}}^{n-1} \frac{m^{-2} \bar{D}_{\mu} \bar{D}^{\mu}+\alpha_{p^{\prime}}^{2}}{\alpha_{p}^{2}-\alpha_{p^{\prime}}^{2}} \phi, \quad p=0, \ldots, n-1$

Let us notice that $\left(J_{p}\right)_{\mu}$ are the linear combinations of $\left(J_{p}\right)_{i}$ in (63). We deal with objects $\left(J_{p}\right)_{\mu}$ for reasons of convenience.

The $n+N$-parameter series of interaction vertices (56) for free equations (76) read

$$
\begin{aligned}
E & \equiv m^{2} \prod_{p=0}^{n-1}\left(m^{-2} \bar{D}_{\mu}(\beta, B) \bar{D}^{\mu}(\beta, B)+\alpha_{p}^{2}\right) \phi=0, \\
\mathcal{E}_{\mu} & \equiv \frac{m^{2}}{2} \sum_{q=1}^{N} A_{q}\left(\mathcal{W}^{q}\right)_{\mu \nu} \Phi^{\nu} \\
& +i \sum_{p=0}^{n-1} \beta_{p}\left(\phi^{*(p)} \bar{D}_{\mu}(\beta, B) \phi^{(p)}-\phi^{(p)} \bar{D}_{\mu}(\beta, B) \phi^{*(p)}\right)=0,
\end{aligned}
$$

\footnotetext{
${ }^{4}$ The fields $\phi^{(p)}$ describe irreducible components of reducible representation of the Poincare group that is described by the higher derivative theory of complex scalar. The vectors $\left(J_{p}\right)_{\mu}, p=0, \ldots, n-1$, are charge currents of irreducible components, while the coupling constants $\beta_{p}, p=0, \ldots, n-1$, can be interpreted as the charges of components.
} 
where $\bar{D}_{\mu}(\beta, B)$ is the covariant derivative $(77), \phi^{(p)}$ is given by (80), and $\beta, \beta_{p}, B_{q}, p=0, \ldots, n-1, q=1, \ldots, N-1$, are the coupling parameters.

Equations (81) are obviously gauge invariant with respect to gauge symmetry (74). The gauge identity between Eq. (81) is seen in the form

$$
\partial^{\mu} \mathcal{E}_{\mu}-i \sum_{p=0}^{n-1} \prod_{\substack{p^{\prime}=0 \\ p^{\prime} \neq p}}^{n-1} \frac{\beta_{p}}{\alpha_{p}^{2}-\alpha_{p^{\prime}}^{2}}\left(\phi^{*(p)} E-\phi^{(p)} E^{*}\right)=0 .
$$

The series of the energy-momentum tensors (52) for nonlinear theory (81) has the structure

$\boldsymbol{\Theta}_{\mu \nu}(\phi, \Phi)=\sum_{p=0}^{n-1} \beta_{p}\left(T_{p}\right)_{\mu \nu}(\phi, \Phi)+\sum_{q=0}^{N-1} B_{q}\left(T_{q}\right)_{\mu \nu}(\Phi)$,

where the quantities $\left(T_{p}\right)_{\mu \nu}, p=0, \ldots, n-1$, and $\left(T_{q}\right)_{\mu \nu}$, $q=0, \ldots, N-1$, read

$$
\begin{aligned}
\left(T_{p}\right)_{\mu \nu}(\phi, \Phi)= & \bar{D}_{\mu} \phi^{*(p)} \bar{D}_{\nu} \phi^{(p)}+\bar{D}_{\nu} \phi^{*(p)} \bar{D}_{\mu} \phi^{(p)} \\
& -\eta_{\mu \nu} \bar{D}_{\lambda} \phi^{*(p)} \bar{D}^{\lambda} \phi^{(p)}+m^{2} \alpha_{p}^{2} \eta_{\mu \nu} \phi^{*(p)} \phi^{(p)},
\end{aligned}
$$

$$
\begin{aligned}
\left(T_{q}\right)_{\mu \nu}(\Phi)= & \sum_{r, s=1}^{N-1} \sum_{q^{\prime}=1}^{N} C_{r, s}^{q, q^{\prime}} A_{q^{\prime}}\left(\Phi_{\mu}^{(r)} \Phi_{\nu}^{(s)}+\Phi_{\nu}^{(r)} \Phi_{\mu}^{(s)}\right. \\
& \left.-\eta_{\mu \nu} \Phi^{(r)}{ }_{\lambda} \Phi^{(s) \lambda}\right), \quad \Phi_{\mu}^{(q)} \equiv\left(\mathcal{W}^{q}\right)_{\mu \nu} \Phi^{\nu} .
\end{aligned}
$$

The constants $C_{r, s}^{q, q^{\prime}}$ are defined as follows:

$$
C_{r, s}^{q, q^{\prime}}= \begin{cases}-1, q>q^{\prime}, & q>r, s, \\ & q+q^{\prime}=r+s+1 ; \\ 1, q<q^{\prime}, & q \leq r, s, \\ & q+q^{\prime}=r+s+1 ; \\ \text { 0, otherwise. } & \end{cases}
$$

As is seen from (83), the quantity $\boldsymbol{\Theta}^{\mu \nu}$ is the deformation of selected representative of energy-momentum tensor series of free complex scalar and Chern-Simons,

$$
\begin{aligned}
\Theta_{\mu \nu}^{\mathrm{free}}(\phi, \Phi)= & \sum_{q=0}^{N-1} B_{q}\left(T_{q}\right)_{\mu \nu}(\Phi)+\sum_{p=0}^{n-1} \beta_{p}\left(\partial_{\mu} \phi^{*(p)} \partial_{\nu} \phi^{(p)}\right. \\
& +\partial_{\nu} \phi^{*(p)} \partial_{\mu} \phi^{(p)}-\eta_{\mu \nu} \partial_{\lambda} \phi^{*(p)} \partial^{\lambda} \phi^{(p)} \\
& \left.+m^{2} \alpha_{p}^{2} \eta_{\mu \nu} \phi^{*(p)} \phi^{(p)}\right) .
\end{aligned}
$$

In this formula all the parameters of the conserved quantity are fixed by the interaction, so a single representative of free series of conserved quantities survives at the interacting level.
Let us discuss the stability of the theory (81) at the interacting level. The 00-component of (83) can be written in the form

$$
\begin{aligned}
\boldsymbol{\Theta}_{00}(\phi, \Phi)= & \frac{m^{2}}{2} \sum_{r, s=1}^{N-1} C_{r, s}(A, B) \Phi_{\mu}^{(r)} \Phi_{\mu}^{(s)} \\
& +\sum_{p=0}^{n-1} \beta_{p}\left(\bar{D}_{\mu} \phi^{*(p)} \bar{D}_{\mu} \phi^{(p)}+m^{2} \alpha_{p}^{2} \phi^{*(p)} \phi^{(p)}\right) .
\end{aligned}
$$

where the matrix $C_{r, s}(A, B)$ is defined by

$$
C_{r, s}(A, B)=\sum_{q=0}^{N-1} \sum_{q^{\prime}=1}^{N} C_{r, s}^{q, q^{\prime}} B_{q} A_{q^{\prime}}
$$

The expression (88) is a quadratic form in the variables $\Phi^{(q)}{ }_{\mu}, \phi^{(p)}$. It is bounded if $C_{r, s}(A, B), r, s=1, \ldots, N-1$, is a positive definite matrix, and $\beta_{p}>0, p=0, \ldots, n-1$, are positive numbers. ${ }^{5}$

We consider the stability condition of interacting theory as the natural selection rule for admissible couplings. In the sector of scalar field, it restricts the coupling parameters $\beta_{p}$. In the sector of gauge field, the matrix $C_{r, s}(A, B)$ can be positive definite or indefinite depending on the values of the free model parameters $A_{q}$ and coupling constants $B_{q}$. In the work [27] the following fact is noticed about the free extended Chern-Simons theory: the series of conserved quantities (85) includes the bounded representative if the characteristic polynomial of the theory (76) has simple real nonzero roots and a zero root of multiplicity one or two. In all the other cases, the theory is unstable. For the viewpoint of representation theory, the stability condition requires from the free extended Chern-Simons theory to describe the unitary representation of the Poincaré group. The roots of

\footnotetext{
${ }^{5}$ The quantity $C_{r, s}(A, B)$ is the Bezout matrix of the characteristic polynomial (7) of free extended Chern-Simons theory and characteristic polynomial of derived symmetry (15), (16). It is defined by the relation

$$
\sum_{r, s=1}^{N-1} C_{r, s}(A, B) z^{r} u^{s}=\frac{M(A ; z) N(B ; u)-M(A ; u) N(B ; z)}{z-u},
$$
}

where

$$
M(A ; z)=\sum_{q=1}^{N} A_{q} z^{q}, \quad N(B ; z)=\sum_{q=0}^{N-1} B_{q+1} z^{q+1} .
$$

Accounting this definition, formula (86) takes the form

$$
C_{r, s}^{q, q^{\prime}}=\frac{\partial^{2} C(A, B)}{\partial A_{q} \partial B_{q^{\prime}}} .
$$


characteristic equation determine the masses of irreducible representations in this case. Once the roots of the characteristic equation of extended Chern-Simons theory are such that any conserved quantity is unbounded in the series (85), and therefore the stable interactions are impossible to include, the free theory corresponds to the nonunitary representation. It seems quite natural that the nonunitary theory cannot be stable at interacting level.

In general, the interaction (81) is non-Lagrangian. The Lagrangian interaction vertex corresponds to the following values of coupling parameters:

$\beta=\beta_{0}, \quad B_{1}=\ldots=B_{N}=\beta_{1}=\ldots=\beta_{n-1}=0$.

The conserved quantity for variational couplings is the canonical energy-momentum tensor. As the canonical energy $\left(\boldsymbol{\Theta}_{\text {can }}\right)_{00}$ is unbounded, the Lagrangian interaction is unstable. The field equations (81) include stable interactions, so the corresponding coupling is non-Lagrangian. The coupling parameters that lead to stable interactions are determined by conditions $\beta_{p}>0, p=0, \ldots, n-1$, and positive definiteness requirement for the form $C_{r, s}(A, B)(89)$.

\section{CONCLUSION AND DISCUSSION OF RESULTS}

At first, let us summarize the basic assumptions about their consequences for the class of higher derivative field theories we study in this paper. We consider inclusion of stable interactions in certain class of higher derivative field theories which we term as derived models. The derived higher derivative theory implies that the same field admits free field equation with the wave operator $W$ without higher derivatives. We call this model a primary theory. The wave operator of the derived theory is a $n$th order polynomial in $W$. We call it a characteristic polynomial of the derived theory. Every symmetry of primary theory results in the $n$ parametric series of symmetries of the derived theory. These symmetries are connected to the series of independent conserved quantities. In particular, the translation invariance of the primary model results in the $n$-parametric series of conserved tensors. Under certain assumptions about the roots of characteristic polynomial, the series can include bounded conserved quantities. The canonical energy-momentum is included into the series, though it is always unbounded. Once the free derived theory admits bounded conserved quantities, it is considered stable.

We consider inclusion of interactions between two different derived field theories. One of these is supposed to be gauge invariant, and another one is non-gauge. We assume that the primary theories of these two models admit consistent and stable interactions, with the gauge symmetry remaining Abelian at interacting level. We also impose certain technical restrictions on the interaction between primary theories as explained in the Sec. III. In particular, these restrictions exclude the "gauging" scenarios of inclusion of interactions. ${ }^{6}$ In principle, these restrictions could be relaxed without breaking the general scheme of inclusion of stable interactions in the derived-type theories, though this would make the consideration much more cumbersome. Once the primary theories admit consistent interactions, these can be lifted to the level of derived theory. The lift is not unique, we get $n+N$-parametric series of consistent interactions, where $n, N$ are the orders of characteristic polynomials of the two models. At free level, the two derived theories admitted $n+N$-parametric series of the conserved tensors. Upon inclusion of interaction, only one tensor conserves with any fixed set of the interaction parameters. The canonical energy momentum is conserved if the interaction is Lagrangian. As the canonical energy is unbounded, the Lagrangian interaction is unstable. Once the free derived theory admits bounded conserved quantity, its conservation can be always preserved with appropriate consistent and Poincaré invariant interaction, so the stability persists at interacting level, though with the non-Lagrangian vertex.

Also notice that even if the interaction is included in such a way that none of the bounded conserved quantities (from the series identified in Sec. II) of the free theory remains conserving at interacting level, these nonconserved currents can be still informative. So they can be still relevant for Lagrangian interactions. If the dynamics is known of bounded nonconserved quantity, than we learn about the compact evolving phase-space surface where the dynamics is confined. This might be useful for identifying the isles of stability, and evaluating the velocity of running away solutions. For discussion of these ideas and further references see the recent work [37].

Even though the vertices of stable interactions are always non-Lagrangian in this class of higher derivative theories, they can still admit quantization. The matter is that these non-Lagrangian field equations can admit Lagrange anchor which allows one to quantize the non-Lagrangian field theory. The examples are provided in the paper [26] where the some special interactions are considered between the derived model with the quadratic characteristic polynomial, and the theory without higher derivatives. Another side of the existence of the Lagrange anchor is that the theory should admit Hamiltonian formalism even though the higher derivative equations are non-Lagrangian. The explicit examples of Hamiltonian formalism for third-order non-Lagrangian derived systems can be found in papers $[28,29]$. The constrained Hamiltonian formalism for $n$-th order derived theory with the stable interactions constructed in Sec. IV is developed in the recent work [38].

\footnotetext{
${ }^{6}$ The "gauging" schemes mean that the gauge symmetry of self-interacting gauge fields can change upon inclusion of the non-gauge matter fields. Concerning the literature on gauging, we can mention $[35,36]$ and references therein.
} 


\section{ACKNOWLEDGMENTS}

We thank P. Kazinski, A. Kamenshchik, A. Sharapov, and A. Vikman for discussions. The work of V. A. A. is supported by Tomsk State University Competitiveness Improvement Program. The work of D. S. C. is supported by the Russian Science Foundation Grant No. 18-72-10123 in association with the Lebedev Physical Institute of RAS. The work of S. L. L. is supported by the Project No. 3.5204.2017/6.7 of Russian Ministry of Science and Education.

\section{APPENDIX A: THE PROOF OF IDENTITIES (62)}

Let us mention at first the obvious fact we make use of below. The anticommutator $\{$,$\} of self-adjoint and anti-$ self-adjoint operators is anti-self-adjoint. This means, the diagonal elements vanish of the anticommutator

$$
\left\langle\phi,\left\{\bar{W}^{p}, R_{\alpha}\right\} \phi\right\rangle \equiv 0, \quad p=0,1,2 \ldots
$$

If the space-time translation, being the anti-self-adjoint operator, is substituted in this relation, the diagonal element will vanish. This means, we get the integral of a total divergence

$$
\left\langle\phi,\left\{\bar{W}^{p}, \partial^{\mu}\right\} \phi\right\rangle=\int d^{d} x \partial_{\nu} \Sigma_{p}^{\mu \nu}(\phi, \Phi), \quad p=0,1,2 \ldots,
$$

with $\Sigma_{p}^{\mu \nu}$ being some second-rank tensor.

The relation (62.a)) is proved by induction. The statement is obviously true for $p=0$. Assuming that (62.a)) holds for some unspecified value of $p=k$ and using (40), we find

$$
\begin{aligned}
\delta_{\varepsilon}\left(\bar{W}^{k+1} \phi\right)^{a} & =\beta \varepsilon^{\alpha}\left(\bar{W}_{c}^{a} R_{b \alpha}^{c}+R_{\alpha}^{i} \partial_{i} \Gamma_{b}^{a}\right)\left(\bar{W}^{k} \phi\right)^{b} \\
& =\beta \varepsilon^{\alpha} R_{b \alpha}^{a}\left(\bar{W}^{k+1} \phi\right)^{b} .
\end{aligned}
$$

As $k$ is arbitrary positive number, the statement holds for any nonnegative $p=0,1,2, \ldots$.

Consider relation (62.b). Using the Leibnitz rule to compute variational derivative, we can represent the quantity $\left(\bar{J}_{p}\right)_{i}$ in the following form

$$
\left(\bar{J}_{p}\right)_{i}=\frac{1}{2} \sum_{k=0}^{n} \sum_{l=1}^{p+k} \alpha_{k}\left\langle\phi, \bar{W}^{l-1} \overline{\partial_{i} \Gamma} \bar{W}^{p+k-l} \phi\right\rangle .
$$

Contracting this relation with the gauge generator $R_{\alpha}^{i}$, we get

$$
R_{\alpha}^{i}\left(\bar{J}_{p}\right)_{i}=\frac{1}{2} \sum_{k=0}^{n} \sum_{l=1}^{p+k} \alpha_{k}\left\langle\phi, \bar{W}^{l-1} R_{\alpha}^{i} \overline{\partial_{i} \Gamma} \bar{W}^{p+k-l} \phi\right\rangle .
$$

With the account of relations (A1) and equations of motion (56), we see that

$$
\begin{aligned}
R_{a b \alpha}\left(\bar{W}^{p}\right)^{b}{ }_{c} \phi^{c} E^{a} & =\left\langle\phi, \bar{M} \bar{W} R_{\alpha} \phi\right\rangle \\
& =\frac{1}{2} \sum_{k=0}^{n} \alpha_{k}\left\langle\phi,\left[\bar{W}^{p+k}, R_{\alpha}\right] \phi\right\rangle .
\end{aligned}
$$

The latter quantity can be expressed as a sum of commutators,

$$
\left[R_{\alpha}, \bar{W}^{p+k}\right]=\sum_{l=1}^{p+k} \bar{W}^{l-1}\left[R_{\alpha}, \bar{W}\right] \bar{W}^{p+k-l} .
$$

Substituting this expression into (A6) and adding the result to (A5), we obtain

$$
\begin{aligned}
& R_{\alpha}^{i}\left(\bar{J}_{p}\right)_{i}+R_{a b \alpha}\left(\bar{W}^{p}\right)_{c}^{b} \phi^{c} E^{a} \\
& \quad=\frac{1}{2} \sum_{k=0}^{n} \sum_{l=1}^{p+k} \alpha_{k}\left\langle\phi, \bar{W}^{l-1}\left(\left[\bar{W}, R_{\alpha}\right]+R^{i} \partial_{i} \Gamma\right) \bar{W}^{p+k-l} \phi\right\rangle .
\end{aligned}
$$

Due to identity (41), this expression vanishes identically. This proves the formula (62.b).

Now consider the issue of gauge invariance of currentlike term $\left(\bar{J}_{p}\right)_{i}$. Computing the gauge variation of the expression (A5) and using formula (62.a), we obtain

$$
\begin{aligned}
\delta_{\varepsilon}\left(\bar{J}_{p}\right)_{i}= & \frac{1}{2} \beta \varepsilon^{\alpha} \sum_{k=0}^{n} \sum_{l=1}^{p+k} \alpha_{k}\left\langle\phi, \bar{W}^{l-1} \partial_{i}\left(\left[\bar{W}, R_{\alpha}\right]\right.\right. \\
& \left.\left.+R^{j}{ }_{\alpha} \partial_{j} \Gamma\right) \bar{W}^{p+k-l} \phi\right\rangle .
\end{aligned}
$$

Again, this expression vanishes identically as a result of identity (41). This proves formula (62.c).

\section{APPENDIX B: EXISTENCE AND STRUCTURE OF SECOND-RANK CONSERVED TENSOR $\Theta^{\mu \nu}(\beta, B)$}

In this Appendix, we prove that the rhs of Eq. (61) is the conserved tensor. Substituting the equations of motion (56) into this relation, using the definition of currentlike term (57), we get

$$
\begin{aligned}
& \int d^{d} x \partial_{\nu} \Theta^{\mu \nu}(\beta, B) \\
& =\sum_{k=0}^{n} \sum_{p=0}^{n-1} \alpha_{k} \beta_{p}\left\langle\phi,\left(\partial^{\mu} \bar{W}^{p+k}+\frac{1}{2} \bar{\Phi}^{i} \partial_{i} \partial^{\mu} \bar{W}^{p+k}\right) \phi\right\rangle \\
& \quad+\sum_{q=0}^{N-1} B_{q}\left\langle\Phi, \partial^{\mu} \mathcal{M} \Phi\right\rangle .
\end{aligned}
$$


The second term defines the series of energy-momentum tensors of free theory of gauge field (53),

$$
\begin{aligned}
\partial_{\nu} T^{\mu \nu}(\Phi) & =\sum_{q=0}^{N-1} B_{q} \Phi^{i}\left(\partial^{\mu} \mathcal{M} \Phi\right)_{i}, \\
T^{\mu \nu}(\Phi) & =\sum_{q=0}^{N-1} B_{q} T_{q}^{\mu \nu}(\Phi) .
\end{aligned}
$$

It remains to prove that the first term is a divergence of some tensor. Integrating by parts, we decompose the entries of the sum into three contributions

$$
\begin{aligned}
& \frac{1}{2}\left\{-\left\langle\phi,\left(\left[\bar{W}^{p+k}, \partial^{\mu}\right]+\partial^{\mu} \bar{\Phi}^{i} \partial_{i} \bar{W}^{p+k}\right) \phi\right\rangle+\left\langle\phi,\left\{\bar{W}^{p+k}, \partial^{\mu}\right\} \phi\right\rangle\right. \\
& \left.\quad+\int d^{d} x \partial^{\mu}\left\langle\phi, \bar{\Phi}^{i} \partial_{i} \bar{W}^{p+k} \phi\right\rangle\right\},
\end{aligned}
$$

where the square brackets [,] and braces $\{$,$\} denote the$ commutator and anticommutator of enclosed operators. The first term vanishes identically due to (42). The second term is the total divergence (A2), as well as the third one. The consistency of formula (61) is proven.
The conserved tensor $\boldsymbol{\Theta}^{\mu \nu}(\beta, B)$ has the following structure:

$$
\begin{aligned}
\Theta^{\mu \nu}(\beta, B)= & \sum_{k=0}^{n} \sum_{p=0}^{n-1} \alpha_{k} \beta_{p}\left(\Sigma_{p+k}^{\mu \nu}(\phi, \Phi)\right. \\
& \left.+\frac{1}{2} \eta^{\mu \nu}\left\langle\phi, \bar{\Phi}^{i} \partial_{i} \bar{W}^{p+k} \phi\right\rangle\right)+\sum_{q=0}^{N-1} B_{q} T_{q}^{\mu \nu}(\Phi),
\end{aligned}
$$

where $T_{q}{ }^{\mu \nu}(\Phi)$ are the energy-momentum tensors of free gauge field, and $\Sigma_{p+k}{ }^{\mu \nu}(\phi, \Phi)$ is defined in (A2). As in the free limit

$T_{p}^{\mu \nu}(\phi)=\sum_{k=0}^{n} \alpha_{k} \Sigma_{p+k}^{\mu \nu}, \quad\left\langle\phi, \bar{\Phi}^{i} \partial_{i} \bar{W}^{p+k} \phi\right\rangle=0$,

the quantity $\Theta^{\mu \nu}(\beta, B)$ is a deformation of an exclusive representative in the series of energy-momentum tensors (53).
[1] A. Pais and G. E. Uhlenbeck, On field theories with nonlocalized action, Phys. Rev. 79, 145 (1950).

[2] M. Pavsic, Pais-Uhlenbeck oscillator and negative energies, Int. J. Geom. Methods Mod. Phys. 13, 1630015 (2016).

[3] R. P. Woodard, The theorem of Ostrogradsky, Scholarpedia 10, 32243 (2015).

[4] A. V. Smilga, Classical and quantum dynamics of higherderivative systems, Int. J. Mod. Phys. A 32, 1730025 (2017).

[5] A. Salvio, Quadratic gravity, Front. Phys. 6, 77 (2018).

[6] E. T. Tomboulis, Renormalization and unitarity in higher derivative and nonlocal gravity theories, Mod. Phys. Lett. A 30, 1540005 (2015).

[7] A. Belenchia, M. Letizia, S. Liberati, and E. Di Casola, Higher-order theories of gravity: Diagnosis, extraction and reformulation via non-metric extra degrees of freedom-a review, Rep. Prog. Phys. 81, 036001 (2018).

[8] T. P. Sotiriou and V. Faraoni, $f(R)$ theories of gravity, Rev. Mod. Phys. 82, 451 (2010).

[9] A. De Felice and S. Tsujikawa, $f(R)$ theories, Living Rev. Relativity 13, 3 (2010).

[10] A. V. Smilga, Benign vs malicious ghosts in higher-derivative theories, Nucl. Phys. B706, 598 (2005).

[11] E. A. Bergshoeff, M. Kovacevic, J. Rosseel, P. K. Townsend, and Y. Yin, A spin-4 analog of $3 D$ massive gravity, Classical Quantum Gravity 28, 245007 (2011).
[12] M. Nitta and R. Yokokura, Topological couplings in higher derivative extensions of supersymmetric three-form gauge theories, arXiv:1810.12678.

[13] K. Andrzejewski, K. Bolonek, J. Gonera, and P. Maslanka, Canonical formalism and quantization of perturbative sector of higher-derivative theories, Phys. Rev. A 76, 032110 (2007).

[14] A. V. Smilga, Comments on the dynamics of the PaisUhlenbeck oscillator, SIGMA 5, 017 (2009).

[15] T. Chen, M. Fasiello, E. A. Lim, and A. J. Tolley, Higher derivative theories with constraints: Exorcising Ostrogradskis ghost, J. Cosmol. Astropart. Phys. 02 (2013) 042.

[16] M. Pavsic, Stable self-interacting Pais-Uhlenbeck oscillator, Mod. Phys. Lett. A 28, 1350165 (2013).

[17] M. Avendao-Camacho, J. A. Vallejo, and Yu. Vorobiev, A perturbation theory approach to the stability of the PaisUhlenbeck oscillator, J. Math. Phys. 58, 093501 (2017).

[18] B. Podolsky, A generalized electrodynamics. Part I-nonquantum, Phys. Rev. 62, 68 (1942).

[19] S. Deser and R. Jackiw, Higher derivative Chern-Simons extensions, Phys. Lett. B 451, 73 (1999).

[20] R. Bufalo, B. M. Pimentel, and G. E. R. Zambrano, Renormalizability of generalized quantum electrodynamics, Phys. Rev. D 86, 125023 (2012).

[21] M. Ghasemkhani and R. Bufalo, Higher derivative ChernSimons extension in the noncommutative $\mathrm{QED}_{3}$, Phys. Rev. D 91, 125013 (2015). 
[22] A. A. Nogueira, C. Palechor, and A. F. Ferrari, Reduction of order and Fadeev-Jackiw formalism in generalized electrodynamics, Nucl. Phys. B939, 372 (2019).

[23] H. Lu, Y. Pang, and C. N. Pope, Conformal gravity and extensions of critical gravity, Phys. Rev. D 84, 064001 (2011).

[24] H. Lu, Y. Pang, and C. N. Pope, Black holes in sixdimensional conformal gravity, Phys. Rev. D 87, 104013 (2013).

[25] J. Maldacena, Einstein gravity from conformal gravity, arXiv:1105.5632.

[26] D. S. Kaparulin, S. L. Lyakhovich, and A. A. Sharapov, Classical and quantum stability of higher-derivative dynamics, Eur. Phys. J. C 74, 3072 (2014).

[27] D. S. Kaparulin, I. Yu. Karataeva, and S. L. Lyakhovich, Higher derivative extensions of 3d Chern-Simons models: Conservation laws and stability, Eur. Phys. J. C 75, 552 (2015).

[28] V. A. Abakumova, D. S. Kaparulin, and S. L. Lyakhovich, Multi-Hamiltonian formulations and stability of higherderivative extensions of $3 \mathrm{~d}$ Chern-Simons, Eur. Phys. J. C 78, 115 (2018).

[29] D. S. Kaparulin, I. Yu. Karataeva, and S. L. Lyakhovich, Third order extensions of $3 d$ Chern-Simons interacting to gravity: Hamiltonian formalism and stability, Nucl. Phys. B934, 634 (2018).

[30] D. S. Kaparulin, S. L. Lyakhovich, and A. A. Sharapov, Stable interactions via proper deformations, J. Phys. A 49, 155204 (2016).
[31] P. O. Kazinski, S. L. Lyakhovich, and A. A. Sharapov, Lagrange structure and quantization, J. High Energy Phys. 07 (2005) 076.

[32] D. S. Kaparulin, S. L. Lyakhovich, and A. A. Sharapov, Rigid symmetries and conservation laws in non-Lagrangian field theory, J. Math. Phys. 51, 082902 (2010).

[33] B. DeWitt, Dynamical Theory of Groups and Fields (Gordon and Breach, New York, 1965).

[34] D. S. Kaparulin, S. L. Lyakhovich, and A. A. Sharapov, Consistent interactions and involution, J. High Energy Phys. 01 (2013) 097.

[35] M. Trigiante, Gauged supergravities, Phys. Rep. 680, 1 (2017).

[36] G. Barnich, N. Boulanger, M. Henneaux, B. Julia, V. Lekeu, and A. Ranjbar, Deformations of vector-scalar models, J. High Energy Phys. 02 (2018) 064; G. Barnich and N. Boulanger, A note on local BRST cohomology of Yang-Mills type theories with free Abelian factors, J. Math. Phys. 59, 052302 (2018).

[37] N. Boulanger, F. Buisseret, F. Dierick, and O. White, Higher-derivative harmonic oscillators: Stability of classical dynamics and adiabatic invariants, Eur. Phys. J. C 79, 60 (2019).

[38] V. A. Abakumova, D. S. Kaparulin, and S. L. Lyakhovich, Stable interactions between extended Chern-Simons theory and charged scalar field with higher derivatives: Hamiltonian formalism, Russ. Phys. J. 62 (2019). 